\title{
Compact McEliece Keys from Goppa Codes
}

\author{
Rafael Misoczki and Paulo S.L.M. Barreto* \\ Departamento de Engenharia de Computação e Sistemas Digitais (PCS), \\ Escola Politécnica, Universidade de São Paulo, Brazil \\ \{rmisoczki, pbarreto\}@larc.usp.br
}

\begin{abstract}
The classical McEliece cryptosystem is built upon the class of Goppa codes, which remains secure to this date in contrast to many other families of codes but leads to very large public keys. Previous proposals to obtain short McEliece keys have primarily centered around replacing that class by other families of codes, most of which were shown to contain weaknesses, and at the cost of reducing in half the capability of error correction. In this paper we describe a simple way to reduce significantly the key size in McEliece and related cryptosystems using a subclass of Goppa codes, while also improving the efficiency of cryptographic operations to $\tilde{O}(n)$ time, and keeping the capability of correcting the full designed number of errors in the binary case.
\end{abstract}

\section{Introduction}

Quantum computers can potentially break most if not all conventional cryptosystems actually deployed in practice, namely, all systems based on the integer factorization problem (like RSA) or the discrete logarithm problem (like traditional or elliptic curve Diffie-Hellman and DSA, and also all of pairing-based cryptography).

Certain classical cryptosystems, inspired on computational problems of a nature entirely different from the above and potentially much harder to solve, remain largely unaffected by the threat of quantum computing, and have thus been called quantum-resistant or, more suggestively, 'post-quantum' cryptosystems. These include lattice-based cryptosystems and syndrome-based cryptosystems like McEliece [16] and Niederreiter [19]. Such systems usually have even a speed advantage over conventional schemes; for instance, both McEliece and Niederreiter encryption over a code of length $n$ has time complexity $O\left(n^{2}\right)$, while Diffie-Hellman/DSA and (private exponent) RSA with $n$-bit keys have time complexity $O\left(n^{3}\right)$. On the other hand, they are plagued by very large keys compared to their conventional counterparts.

It is therefore of utmost importance to seek ways to reduce the key sizes for post-quantum cryptosystems while keeping their security level. The first steps

\footnotetext{
* Supported by the Brazilian National Council for Scientific and Technological Development (CNPq) under research productivity grant 312005/2006-7 and universal grant 485317/2007-9, and by the Science Foundation Ireland (SFI) as E. T. S. Walton Award fellow under grant 07/W.1/I1824.
} 
toward this goal were taken by Monico et al. using low density parity-check codes [18], by Gaborit using quasi-cyclic codes [8], and by Baldi and Chiaraluce using a combination of both 1 .

However, these proposals were all shown to contain weaknesses [22. In those proposals the trapdoor is protected essentially by no other means than a private permutation of the underlying code. The attack strategy consists of obtaining a solvable system of linear equations that the components of the permutation matrix must satisfy, and was successfully mounted due to the very constrained nature of the secret permutation (since it has to preserve the quasi-cyclic structure of the result) and the fact that the secret code is a subcode of a public code.

A dedicated fix to the problems in [1] is proposed in [2]. More recently, Berger et al. 3] showed how to circumvent the drawbacks of Gaborit's original scheme and remove the weaknesses pointed out in [22] by means of two techniques:

1. Extracting block-shortened public codes from very large private codes, exploiting Wieschebrink's theorem on the NP-completeness of distinguishing punctured codes 29];

2. Working with subfield subcodes over an intermediate subfield between the base field and the extension field of the original code.

These two techniques were successfully applied to quasi-cyclic codes, yet we will see that their applicability is not restricted to that class.

Our contribution: In this paper we propose the class of quasi-dyadic Goppa codes, which admit a very compact parity-check or a generator matrix representation, for efficiently instantiating syndrome-based cryptosystems. We stress that we are not proposing any new cryptosystem, but rather a technique to obtain efficient parameters and algorithms for such systems, current or future. In contrast to many other proposed families of codes [101122 27], Goppa codes have withstood cryptanalysis quite well, and despite considerable progress in the area 1426 , (see also [6] for a survey) they remain essentially unscathed since they were suggested with the very first syndrome-based cryptosystem known, namely, the original McEliece scheme. Our method produces McEliece-type keys that are up to a factor $t=\tilde{O}(n)$ smaller than keys produced from generic $t$-error correcting Goppa codes of length $n$ in characteristic 2 . In the binary case it also retains the ability of correcting the full designed number of errors rather than just half as many, a feature that is missing in all previous attempts at constructing compact codes for cryptographic purposes, including [3. Moreover, the complexity of all typical cryptographic operations become $\tilde{O}(n)$; specifically, under the common cryptographic setting $t=O(n / \lg n)$, code generation, encryption and decryption all have asymptotic complexity $O(n \lg n)$.

The remainder of this paper is organized as follows. Section 2 introduces some basic concepts of coding theory. In section 3 we describe our proposal of using binary Goppa codes in quasi-dyadic form, and how to build them. We consider hardness issues in Section 4 , and efficiency issues, including guidelines on how to choose parameters, in Section 5 . We conclude in Section 6. 


\section{Preliminaries}

In what follows all vector and matrix indices are numbered from zero onwards.

Definition 1. Given a ring $\mathcal{R}$ and a vector $h=\left(h_{0}, \ldots, h_{n-1}\right) \in \mathcal{R}^{n}$, the dyadic matrix $\Delta(h) \in \mathcal{R}^{n \times n}$ is the symmetric matrix with components $\Delta_{i j}=h_{i \oplus j}$ where $\oplus$ stands for bitwise exclusive-or on the binary representations of the indices. The sequence $h$ is called its signature. The set of dyadic $n \times n$ matrices over $\mathcal{R}$ is denoted $\Delta\left(\mathcal{R}^{n}\right)$. Given $t>0, \Delta(t, h)$ denotes $\Delta(h)$ truncated to its first $t$ rows.

One can recursively characterize a dyadic matrix when $n$ is a power of 2 : any $1 \times 1$ matrix is dyadic, and for $k>0$ any $2^{k} \times 2^{k}$ dyadic matrix $M$ has the form

$$
M=\left[\begin{array}{ll}
A & B \\
B & A
\end{array}\right]
$$

where $A$ and $B$ are $2^{k-1} \times 2^{k-1}$ dyadic matrices. It is not hard to see that the signature of a dyadic matrix coincides with its first row. Dyadic matrices form a commutative subring of $\mathcal{R}^{n \times n}$ as long as $\mathcal{R}$ is commutative [12.

Definition 2. A dyadic permutation is a dyadic matrix $\Pi^{i} \in \Delta\left(\{0,1\}^{n}\right)$ whose signature is the $i$-th row of the identity matrix.

A dyadic permutation is clearly an involution, i.e. $\left(\Pi^{i}\right)^{2}=I$. The $i$-th row (or equivalently the $i$-th column) of the dyadic matrix defined by a signature $h$ can be written $\Delta(h)_{i}=h \Pi^{i}$.

Definition 3. A quasi-dyadic matrix is a (possibly non-dyadic) block matrix whose component blocks are dyadic submatrices.

Quasi-dyadic matrices are at the core of our proposal. We will be mainly concerned with the case $\mathcal{R}=\mathbb{F}_{q}$, the finite field with $q$ (a prime power) elements.

Definition 4. Given two disjoint sequences $z=\left(z_{0}, \ldots, z_{t-1}\right) \in \mathbb{F}_{q}^{t}$ and $L=$ $\left(L_{0}, \ldots, L_{n-1}\right) \in \mathbb{F}_{q}^{n}$ of distinct elements, the Cauchy matrix $C(z, L)$ is the $t \times n$ matrix with elements $C_{i j}=1 /\left(z_{i}-L_{j}\right)$, i.e.

$$
C(z, L)=\left[\begin{array}{ccc}
\frac{1}{z_{0}-L_{0}} & \cdots & \frac{1}{z_{0}-L_{n-1}} \\
\vdots & \ddots & \vdots \\
\frac{1}{z_{t-1}-L_{0}} & \cdots & \frac{1}{z_{t-1}-L_{n-1}}
\end{array}\right]
$$

Cauchy matrices have the property that all of their submatrices are nonsingular [25]. Notice that, in general, Cauchy matrices are not dyadic and vice-versa, although the intersection of these two classes is non-empty in characteristic 2 .

Definition 5. Given $t>0$ and a sequence $L=\left(L_{0}, \ldots, L_{n-1}\right) \in \mathbb{F}_{q}^{n}$, the Vandermonde matrix $\operatorname{vdm}(t, L)$ is the $t \times n$ matrix with elements $V_{i j}=L_{j}^{i}$. 
Definition 6. Given a sequence $L=\left(L_{0}, \ldots, L_{n-1}\right) \in \mathbb{F}_{q}^{n}$ of distinct elements and a sequence $D=\left(D_{0}, \ldots, D_{n-1}\right) \in \mathbb{F}_{q}^{n}$ of nonzero elements, the Generalized Reed-Solomon code $G R S_{r}(L, D)$ is the $[n, k, r]$ linear error-correcting code defined by the parity-check matrix

$$
H=\operatorname{vdm}(r-1, L) \cdot \operatorname{diag}(D) .
$$

An alternant code is a subfield subcode of a Generalized Reed-Solomon code.

Let $p$ be a prime power, let $q=p^{d}$ for some $d$, and let $\mathbb{F}_{q}=\mathbb{F}_{p}[x] / b(x)$ for some irreducible polynomial $b(x) \in \mathbb{F}_{p}[x]$ of degree $d$. Given a code specified by a parity-check matrix $H \in \mathbb{F}_{q}^{t \times n}$, the trace construction derives from it an $\mathbb{F}_{p}$-subfield subcode by writing the $\mathbb{F}_{p}$ coefficients of each $\mathbb{F}_{q}$ component of $H$ onto $d$ successive rows of a parity-check matrix $T_{d}(H) \in \mathbb{F}_{p}^{d t \times n}$ for the subcode. The related co-trace parity-check matrix $T_{d}^{\prime}(H) \in \mathbb{F}_{p}^{d t \times n}$, equivalent to $T_{d}(H)$ by a left permutation, is obtained from $H$ by writing the $\mathbb{F}_{p}$ coefficients of terms of equal degree from all components on a column of $H$ onto successive rows of $T_{d}^{\prime}(H)$.

Thus, given elements $u_{i}(x)=u_{i, 0}+\cdots+u_{i, d-1} x^{d-1} \in \mathbb{F}_{q}=\mathbb{F}_{p}[x] / b(x)$, the trace construction maps a column $\left(u_{0}, \ldots, u_{t-1}\right)^{\top}$ from $H$ to the column $\left(u_{0,0}, \ldots, u_{0, d-1} ; \ldots ; u_{t-1,0}, \ldots, u_{t-1, d-1}\right)^{\top}$ on the trace matrix $T_{d}(H)$, and to the column $\left(u_{0,0}, \ldots, u_{t-1,0} ; \ldots ; u_{0, d-1}, \ldots, u_{t-1, d-1}\right)^{\top}$ on the co-trace matrix $T_{d}^{\prime}(H)$.

Finally, one of the most important families of linear error-correcting codes for cryptographic purposes is that of Goppa codes:

Definition 7. Given a prime power $p, q=p^{d}$ for some $d$, a sequence $L=$ $\left(L_{0}, \ldots, L_{n-1}\right) \in \mathbb{F}_{q}^{n}$ of distinct elements and a polynomial $g(x) \in \mathbb{F}_{q}[x]$ of degree $t$ such that $g\left(L_{i}\right) \neq 0$ for $0 \leqslant i<n$, the Goppa code $\Gamma(L, g)$ over $\mathbb{F}_{p}$ is the alternant code over $\mathbb{F}_{p}$ corresponding to $G R S_{t}(L, D)$ where $D=$ $\left(g\left(L_{0}\right)^{-1}, \ldots, g\left(L_{n-1}\right)^{-1}\right)$, and its minimum distance is at least $2 t+1$.

An irreducible Goppa code in characteristic 2 can correct up to $t$ errors using Patterson's algorithm [23, or slightly more using Bernstein's list decoding method [5], and $t$ errors can still be corrected by suitable decoding algorithms if the generator $g(x)$ is not irreducible1. In all other cases no algorithm is known that can correct more than $t / 2$ errors (or just a few more).

\section{Goppa Codes in Cauchy and Dyadic Form}

A property of Goppa codes that is central to our proposal is that they admit a parity-check matrix in Cauchy form:

${ }^{1}$ For instance, one can equivalently view the binary Goppa code as the alternant code defined by the generator polynomial $g^{2}(x)$, in which case any alternant decoder will decode $t$ errors. We are grateful to Nicolas Sendrier for pointing this out. 
Theorem 1 ([28]). The Goppa code generated by a monic polynomial $g(x)=$ $\left(x-z_{0}\right) \ldots\left(x-z_{t-1}\right)$ without multiple zeros admits a parity-check matrix of the form $H=C(z, L)$, i.e. $H_{i j}=1 /\left(z_{i}-L_{j}\right), 0 \leqslant i<t, 0 \leqslant j<n$.

This theorem (also appearing in [15, Ch. 12, §3, Pr. 5]) is entirely general when one considers the factorization of the Goppa polynomial over its splitting field, in which case a single root of $g$ is enough to completely characterize the code. For simplicity, we will restrict our attention to the case where all roots of that polynomial are in the field $\mathbb{F}_{q}$ itself.

\subsection{Building a Binary Goppa Code in Dyadic Form}

We now show how to build a binary Goppa code that admits a parity-check matrix in dyadic form. To this end we seek a way to construct dyadic Cauchy matrices. The following theorem characterizes all matrices of this kind.

Theorem 2. Let $H \in \mathbb{F}_{q}^{n \times n}$ with $n>1$ be simultaneously a dyadic matrix $H=\Delta(h)$ for some $h \in \mathbb{F}_{q}^{n}$ and a Cauchy matrix $H=C(z, L)$ for two disjoint sequences $z \in \mathbb{F}_{q}^{n}$ and $L \in \mathbb{F}_{q}^{n}$ of distinct elements. Then $\mathbb{F}_{q}$ is a field of characteristic 2, $h$ satisfies

$$
\frac{1}{h_{i \oplus j}}=\frac{1}{h_{i}}+\frac{1}{h_{j}}+\frac{1}{h_{0}},
$$

and $z_{i}=1 / h_{i}+\omega, L_{j}=1 / h_{j}+1 / h_{0}+\omega$ for some $\omega \in \mathbb{F}_{q}$.

Proof. Since a dyadic matrix is symmetric, the sequences that define it must satisfy $1 /\left(z_{i}-L_{j}\right)=1 /\left(z_{j}-L_{i}\right)$, hence $L_{j}=z_{i}+L_{i}-z_{j}$ for all $i$ and $j$. Then $z_{i}+L_{i}$ must be a constant $\alpha$, and taking $i=0$ in particular this simplifies to $L_{j}=\alpha-z_{j}$. Substituting back into the definition $M_{i j}=1 /\left(z_{i}-L_{j}\right)$ one sees that $H_{i j}=1 /\left(z_{i}+z_{j}+\alpha\right)$. But dyadic matrices also have constant diagonal, namely, $H_{i i}=1 /\left(2 z_{i}+\alpha\right)=h_{0}$. This is only possible if all $z_{i}$ are equal (contradicting the definition of a Cauchy matrix), or else if the characteristic of the field is 2, as claimed.

In this case we see that $\alpha=1 / h_{0}$, and hence $H_{i j}=1 /\left(z_{i}+z_{j}+1 / h_{0}\right)$. Plugging in the definition $H_{i j}=h_{i \oplus j}$ we get $1 / H_{i j}=1 / h_{i \oplus j}=z_{i}+z_{j}+1 / h_{0}$, and taking $j=0$ in particular this yields $1 / h_{i}=z_{i}+z_{0}+1 / h_{0}$, or simply $z_{i}=1 / h_{i}+1 / h_{0}+z_{0}$. Substituting back one obtains $1 / h_{i \oplus j}=z_{i}+z_{j}+1 / h_{0}=$ $1 / h_{i}+1 / h_{0}+z_{0}+1 / h_{j}+1 / h_{0}+z_{0}+1 / h_{0}=1 / h_{i}+1 / h_{j}+1 / h_{0}$, as expected.

Finally, define $\omega=1 / h_{0}+z_{0}$ and substitute into the derived relations $z_{i}=$ $1 / h_{i}+1 / h_{0}+z_{0}$ and $L_{j}=\alpha-z_{j}$ to get $z_{i}=1 / h_{i}+\omega$ and $L_{j}=1 / h_{j}+1 / h_{0}+\omega$, as desired.

Therefore all we need is a method to solve Equation 1. The technique we propose consists of simply choosing distinct nonzero $h_{0}$ and $h_{i}$ at random where $i$ scans all powers of two smaller than $n$, and setting all other values as

$$
h_{i+j} \leftarrow \frac{1}{\frac{1}{h_{i}}+\frac{1}{h_{j}}+\frac{1}{h_{0}}}
$$


for $0<j<i$ (so that $i+j=i \oplus j$ ), as long as this value is well-defined. Algorithm 1 captures this idea. Since each element of the signature $h$ is assigned a value exactly once, its running time is $O(n)$ steps. The notation $u \stackrel{\$}{\leftarrow} U$ means that variable $u$ is uniformly sampled at random from set $U$. For convenience we also define the essence of $h$ to be the sequence $\eta_{s}=1 / h_{2^{s}}+1 / h_{0}$ for $s=$ $0, \ldots,\lceil\lg n\rceil-1$ together with $\eta_{\lceil\lg n\rceil}=1 / h_{0}$, so that, for $i=\sum_{k=0}^{\lceil\lg n\rceil-1} i_{k} 2^{k}$, $1 / h_{i}=\eta_{\lceil\lg n\rceil}+\sum_{k=0}^{\lceil\lg n\rceil-1} i_{k} \eta_{k}$.

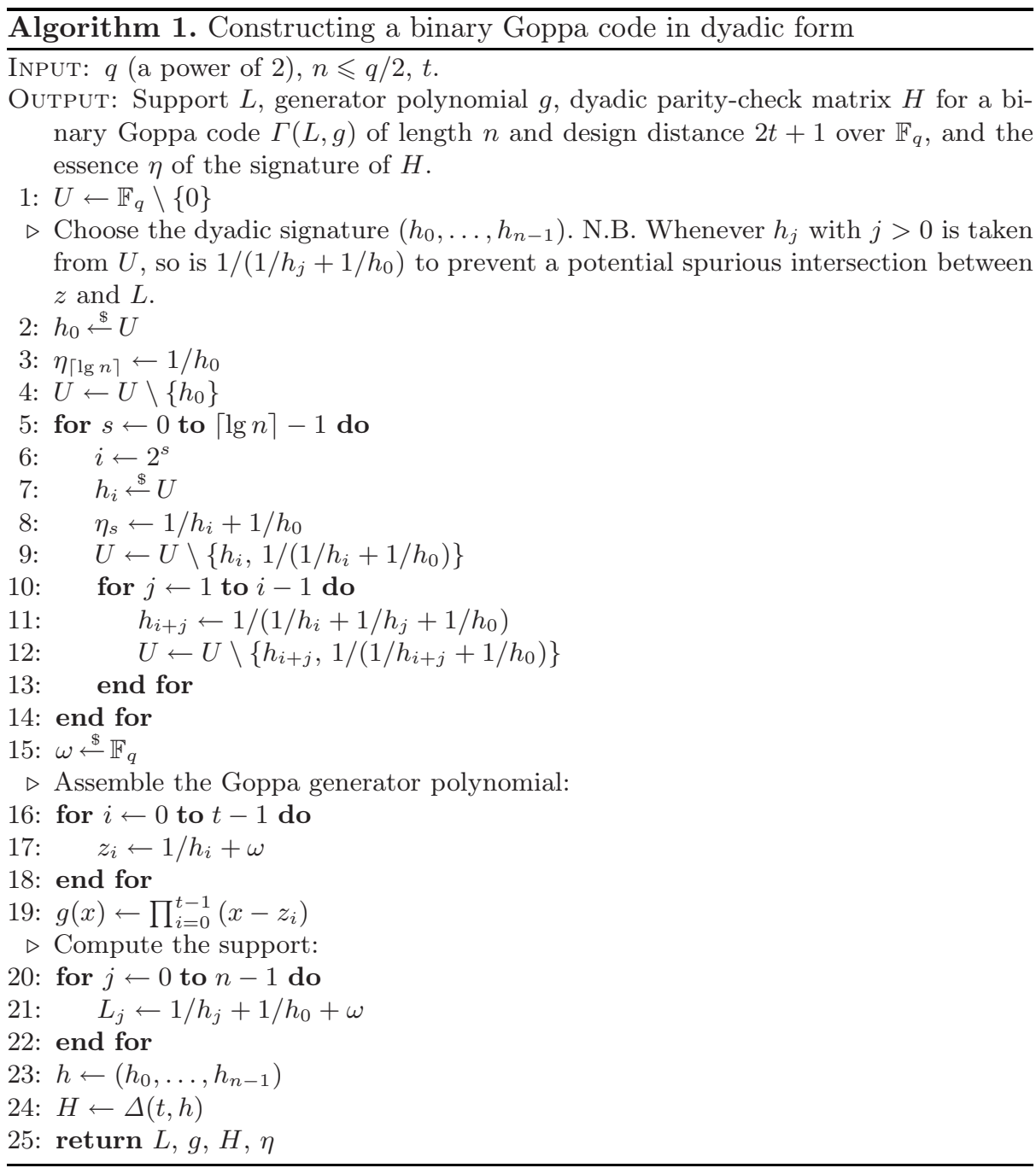

Theorem 3. Algorithm 1 produces up to $\prod_{i=0}^{\lceil\lg n\rceil}\left(q-2^{i}\right)$ Goppa codes in dyadic form. 
Proof. Each dyadic signature produced by Algorithm 1 is entirely determined by the values $h_{0}$ and $h_{2 s}$ for $s=0, \ldots,\lceil\lg n\rceil-1$ chosen at steps 2 and 7 ( $\omega$ only produces equivalent codes). Along the loop at line 5, exactly $2 i=2^{s+1}$ elements are erased from $U$, corresponding to the choices of $h_{2^{s}} \ldots h_{2^{s+1}-1}$. At the end of that loop, $2+2 \sum_{\ell=0}^{s} 2^{\ell}=2^{s+2}$ elements have been erased in total. Hence at the beginning of each step of the loop only $2^{s+1}$ elements had been erased from $U$, i.e. there are $q-2^{s+1}$ elements in $U$ to choose $h_{2^{s}}$ from, and $q-1$ possibilities for $h_{0}$. Therefore this construction potentially yields up to $(q-1) \prod_{s=0}^{\lceil\lg n\rceil-1}\left(q-2^{s+1}\right)=\prod_{i=0}^{\lceil\lg n\rceil}\left(q-2^{i}\right)$ possible codes.

Theorem 3 actually establishes the number of distinct essences of dyadic signatures corresponding to Cauchy matrices. The roots of the Goppa polynomial are completely specified by the first $\lceil\lg t\rceil$ elements of the essence $\eta$ together with $\eta_{\lceil\lg n\rceil}$, namely, $z_{i}=\eta_{\lceil\lg n\rceil}+\sum_{k=0}^{\lceil\lg t\rceil-1} i_{k} \eta_{k}$, disregarding the $\omega$ term which is implicit in the choice of $\eta_{\lceil\lg n\rceil}$. We see that any permutation of the essence elements $\eta_{0}, \ldots, \eta_{\lceil\lg t\rceil-1}$ only changes the order of those roots. Since the Goppa polynomial itself is defined by its roots regardless of their order, the total number of possible Goppa polynomials is therefore $\left(\prod_{i=0}^{\lceil\lg t\rceil}\left(q-2^{i}\right)\right) /\lceil\lg t\rceil ! \approx(q-t)\left(_{\lceil\lg t\rceil}^{q}\right)$.

For $n \approx q / 2$ the number of dyadic codes can be approximated by $q^{m} Q=2^{m^{2}} Q$ where $Q=\prod_{i=1}^{\infty}\left(1-1 / 2^{i}\right) \approx 0.2887881$. We will also see that the number of quasi-dyadic codes, which we describe next and propose for cryptographic applications, is larger than this. Before we proceed, however, it is interesting to notice that one of the reasons the attack proposed in 22 succeeds against certain quasi-cyclic codes, besides the constrained structure of the applied permutation, is that those schemes start from a known BCH or Reed-Solomon code which is unique up to the choice of a primitive element from the underlying finite field. Thus, in those proposals an initial code over $\mathbb{F}_{2^{m}}$ is at best chosen from a set of $O\left(2^{m}\right)$ codes. In comparison, we start from a secret code sampled from a much larger family of $O\left(2^{m^{2}}\right)$ codes. For instance, while those proposals have only $2^{15}$ starting points over $\mathbb{F}_{2^{16}}$, our scheme can sample a family with more than $2^{254}$ codes over the same field. The main protection of the hidden trapdoor is, of course, the block puncturing process and the more complex blockwise permutation of the initial secret code, as detailed next.

\subsection{Constructing Quasi-Dyadic, Permuted Subfield Subcodes}

To complete the construction it is necessary to choose a compact generator matrix for the subfield subcode. Although the parity check matrix $H$ built by Algorithm 1 is dyadic over $\mathbb{F}_{q}$, the usual trace construction leads to a generator of the dual code that most probably violates the dyadic symmetry. However, by representing each field element to a basis of $\mathbb{F}_{q}$ over the subfield $\mathbb{F}_{p}$, one can view $H$ as a superposition of $d=\left[\mathbb{F}_{q}: \mathbb{F}_{p}\right]$ distinct dyadic matrices over $\mathbb{F}_{p}$, and each of them can be stored in a separate dyadic signature.

A cryptosystem cannot be securely defined on a Goppa code specified directly by a parity-check matrix in Cauchy form, since this would immediately reveal 
the Goppa polynomial $g(x)$ : it suffices to solve the overdefined linear system $z_{i}-L_{j}=1 / H_{i j}$ consisting of $t n$ equations in $t+n$ unknowns.

Algorithm 1 generates fully dyadic codes. We now show how to integrate the techniques of Berger et al. with Algorithm 1 so as to build quasi-dyadic subfield subcodes whose parity-check matrix is a non-dyadic matrix composed of blocks of dyadic submatrices. The principle to follow here is to select, permute, and scale the columns of the original parity-check matrix so as to preserve quasidyadicity in the target subfield subcode and the distribution of introduced errors in cryptosystems. A similar process yields a generator matrix in convenient quasidyadic, systematic form.

For the desired security level (see the discussion in Section 5.1), choose $p=2^{s}$ for some $s, q=p^{d}=2^{m}$ for some $d$ with $m=d s$, a code length $n$ and a design number of correctable errors $t$ such that $n=\ell t$ for some $\ell>d$. For simplicity we assume that $t$ is a power of 2 , but the following construction method can be modified to work with other values.

Run Algorithm 1 to produce a code over $\mathbb{F}_{q}$ whose length $N \gg n$ is a large multiple of $t$ not exceeding the largest possible length $q / 2$, so that the constructed $t \times N$ parity-check matrix $\hat{H}$ can be viewed as a sequence of $N / t$ dyadic blocks $\left[B_{0}|\cdots| B_{N / t-1}\right]$ of size $t \times t$ each. Select uniformly at random $\ell$ distinct blocks $B_{i_{0}}, \ldots, B_{i_{\ell-1}}$ in any order from $\hat{H}$, together with $\ell$ dyadic permutations $\Pi^{j_{0}}, \ldots, \Pi^{j_{\ell-1}}$ of size $t \times t$ and $\ell$ nonzero scale factors $\sigma_{0}, \ldots, \sigma_{\ell-1} \in \mathbb{F}_{p}$. Let $\hat{H}^{\prime}=\left[B_{i_{0}} \Pi^{j_{0}}|\cdots| B_{i_{\ell-1}} \Pi^{j_{\ell-1}}\right] \in\left(\mathbb{F}_{q}^{t \times t}\right)^{\ell}$ and $\Sigma=\operatorname{diag}\left(\sigma_{0} I_{t}, \ldots, \sigma_{\ell-1} I_{t}\right) \in$ $\left(\mathbb{F}_{p}^{t \times t}\right)^{\ell \times \ell}$. Compute the co-trace matrix $H^{\prime}=T_{d}^{\prime}\left(\hat{H}^{\prime} \Sigma\right)=T_{d}^{\prime}\left(\hat{H}^{\prime}\right) \Sigma \in\left(\mathbb{F}_{p}^{t \times t}\right)^{d \times \ell}$ and finally the systematic form $H$ of $H^{\prime}$. Notice that, if the systematic form of $T_{d}^{\prime}\left(\hat{H}^{\prime}\right)$ is $H_{0}$, then $H=U^{-1} H_{0} V$ where $U=\operatorname{diag}\left(\sigma_{0} I_{t}, \ldots, \sigma_{\ell-d-1} I_{t}\right)$ and $V=\operatorname{diag}\left(\sigma_{\ell-d} I_{t}, \ldots, \sigma_{\ell-1} I_{t}\right)$.

The resulting parity-check matrix defines a code of length $n$ and dimension $k=n-d t$ over $\mathbb{F}_{p}$, and since all block operations performed during the Gaussian elimination are carried out in the ring $\Delta\left(\mathbb{F}_{p}^{t}\right)$, the result still consists of dyadic submatrices which can be represented by a signature of length $t$. Hence the whole matrix can be stored in an area a factor $t$ smaller than a general matrix. However, the dyadic submatrices that appear in this process are not necessarily nonsingular, as they are not associated to a Cauchy matrix anymore; should all the submatrices on a column be found to be singular (above or below the diagonal, according to the direction of this process) so that no pivot is possible, the whole block containing that column may be replaced by another block $B_{j^{\prime}}$ chosen at random from $\hat{H}$ as above.

The trapdoor information consisting of the essence $\eta$ of $h$, the sequence $\left(i_{0}, \ldots, i_{\ell-1}\right)$ of blocks, the sequence $\left(j_{0}, \ldots, j_{\ell-1}\right)$ of dyadic permutation identifiers, and the sequence of scale factors $\left(\sigma_{0}, \ldots, \sigma_{\ell-1}\right)$, relates the public code defined by $H$ with the private code defined by $\hat{H}$. The space occupied by the trapdoor information is thus $m^{2}+\ell \lg N+\ell s$ bits. If one starts with the largest possible $N=2^{m-1}$, this simplifies to the maximal size of $m^{2}+\ell(m-1+s)$ bits.

The total space occupied by the essential part of the resulting generator (or parity-check) matrix over $\mathbb{F}_{p}$ is $d t \times(n-d t) / t=d k \mathbb{F}_{p}$ elements, or $m k$ bits - a 
factor $t$ better than plain Goppa codes, which occupy $k(n-k)=m k t$ bits. Had $t$ not been chosen to be a power of 2 , say, $t=2^{u} v$ where $v>1$ is odd, the cost of multiplying $t \times t$ matrices would be in general $O\left(2^{u} u v^{3}\right)$ rather than simply $O\left(2^{u} u\right)$, and the final parity-check matrix would be compressed by only a factor $2^{u}$.

For each code produced by Algorithm 1] the number of codes generated by this construction is $\left(\begin{array}{c}N / t \\ \ell\end{array}\right) \times \ell ! \times t^{\ell} \times(r-1)^{\ell}$, hence $\left(\begin{array}{c}N / t \\ \ell\end{array}\right) \times \ell ! \times t^{\ell} \times(r-1)^{\ell} \times$ $\prod_{i=0}^{\lceil\lg N\rceil}\left(q-2^{i}\right)$ codes are possible in principle.

\subsection{A Toy Example}

Let $\mathbb{F}_{2^{5}}=\mathbb{F}_{2}[u] /\left(u^{5}+u^{2}+1\right)$. The dyadic signature

$$
h=\left(u^{20}, u^{3}, u^{6}, u^{28}, u^{9}, u^{29}, u^{4}, u^{22}, u^{12}, u^{5}, u^{10}, u^{2}, u^{24}, u^{26}, u^{25}, u^{15}\right)
$$

and the offset $\omega=u^{21}$ define a 2-error correcting binary Goppa code of length $N=16$ with $g(x)=\left(x-u^{12}\right)\left(x-u^{15}\right)$ and support $L=$ $\left(u^{21}, u^{29}, u^{19}, u^{26}, u^{6}, u^{16}, u^{7}, u^{5}, u^{25}, u^{3}, u^{11}, u^{28}, u^{27}, u^{9}, u^{22}, u^{2}\right)$. The associated parity-check matrix built according to Theorem 1 is

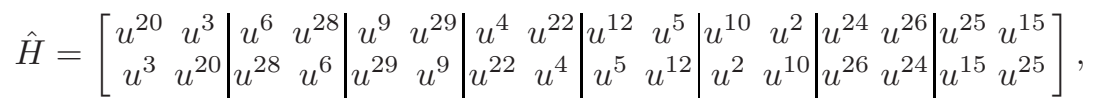

with eight $2 \times 2$ blocks $B_{0}, \ldots, B_{7}$ as indicated. From this we extract the shortened, rearranged and permuted sequence $\hat{H}^{\prime}=\left[B_{7} \Pi^{0}\left|B_{5} \Pi^{1}\right| B_{1} \Pi^{0}\left|B_{2} \Pi^{1}\right|\right.$ $B_{3} \Pi^{0}\left|B_{6} \Pi^{1}\right| B_{4} \Pi^{0}$ ] (because in this example the subfield is the base field itself, all scale factors have to be 1$)$, i.e.:

$$
\hat{H}=\left[\begin{array}{cc|cc|cc|cc|cc|cc|cc}
u^{25} & u^{15} & u^{2} & u^{10} & u^{6} & u^{28} & u^{29} & u^{9} & u^{4} & u^{22} & u^{26} & u^{24} & u^{12} & u^{5} \\
u^{15} & u^{25} & u^{10} & u^{2} & u^{28} & u^{6} & u^{9} & u^{29} & u^{22} & u^{4} & u^{24} & u^{26} & u^{5} & u^{12}
\end{array}\right],
$$

whose co-trace matrix over $\mathbb{F}_{2}$ has the systematic form:

$$
H=\left[\begin{array}{llll|llllllllll}
0 & 1 & 0 & 1 & 1 & 0 & 0 & 0 & 0 & 0 & 0 & 0 & 0 & 0 \\
1 & 0 & 1 & 0 & 0 & 1 & 0 & 0 & 0 & 0 & 0 & 0 & 0 & 0 \\
0 & 1 & 0 & 0 & 0 & 0 & 1 & 0 & 0 & 0 & 0 & 0 & 0 & 0 \\
1 & 0 & 0 & 0 & 0 & 0 & 0 & 1 & 0 & 0 & 0 & 0 & 0 & 0 \\
0 & 0 & 1 & 1 & 0 & 0 & 0 & 0 & 1 & 0 & 0 & 0 & 0 & 0 \\
0 & 0 & 1 & 1 & 0 & 0 & 0 & 0 & 0 & 1 & 0 & 0 & 0 & 0 \\
0 & 1 & 1 & 0 & 0 & 0 & 0 & 0 & 0 & 0 & 1 & 0 & 0 & 0 \\
1 & 0 & 0 & 1 & 0 & 0 & 0 & 0 & 0 & 0 & 0 & 1 & 0 & 0 \\
1 & 1 & 0 & 0 & 0 & 0 & 0 & 0 & 0 & 0 & 0 & 0 & 1 & 0 \\
1 & 1 & 0 & 0 & 0 & 0 & 0 & 0 & 0 & 0 & 0 & 0 & 0 & 1
\end{array}\right]=\left[M^{\top} \mid I_{n-k}\right],
$$

from which one readily obtains the $k \times n=4 \times 14$ generator matrix in systematic form:

$$
G=\left[\begin{array}{llll|llllllllll}
1 & 0 & 0 & 0 & 0 & 1 & 0 & 1 & 0 & 0 & 0 & 1 & 1 & 1 \\
0 & 1 & 0 & 0 & 1 & 0 & 1 & 0 & 0 & 0 & 1 & 0 & 1 & 1 \\
0 & 0 & 1 & 0 & 0 & 1 & 0 & 0 & 1 & 1 & 1 & 0 & 0 & 0 \\
0 & 0 & 0 & 1 & 1 & 0 & 0 & 0 & 1 & 1 & 0 & 1 & 0 & 0
\end{array}\right]=\left[I_{k} \mid M\right],
$$


where both $G$ and $H$ share the essential part $M$ :

$$
M=\left[\begin{array}{llllllllll}
0 & 1 & 0 & 1 & 0 & 0 & 0 & 1 & 1 & 1 \\
1 & 0 & 1 & 0 & 0 & 0 & 1 & 0 & 1 & 1 \\
0 & 1 & 0 & 0 & 1 & 1 & 1 & 0 & 0 & 0 \\
1 & 0 & 0 & 0 & 1 & 1 & 0 & 1 & 0 & 0
\end{array}\right],
$$

which is entirely specified by the elements in boldface and can thus be stored in 20 bits instead of, respectively, $4 \cdot 14=56$ and $10 \cdot 14=140$ bits.

\section{Assessing the Hardness of Decoding Quasi-Dyadic Codes}

The original McEliece (or, for that matter, the original Niederreiter) schemes are perhaps better described as a candidate trapdoor one-way functions rather than full-fledged public-key encryption schemes. Such functions are used in cryptography in many different settings, each with different security requirements, and we do not consider such applications in this paper. Instead we focus purely on the question of inverting the trapdoor function, in other words, decoding.

As we pointed out in Section 1, the well-studied class of Goppa codes remains one of the best choices to instantiate McEliece-like schemes. Although our proposal is ultimately based on Goppa codes, one may wonder whether or not the highly composite nature of the Goppa generator polynomial $g(x)$, or the peculiar structure of the quasi-dyadic parity-check and generator matrices, leak any information that might facilitate decoding without knowledge of the trapdoor.

Yet, any alternant code can be written in Goppa-like fashion by using the diagonal component of its default parity-check matrix (see Definition 6) to interpolate a generating polynomial (not necessarily of degree $t$ ) that is composite with high probability. We are not aware of any way this fact could be used to facilitate decoding without full knowledge of the code structure, and clearly any result in this direction would affect most of the alternant codes proposed for cryptographic purposes to date.

Otmani et al.'s attack against quasi-cyclic codes [22] could be modified to work against Goppa codes in dyadic form. For this reason we adopt the same countermeasures proposed by Berger et al. to thwart it for cyclic codes, namely, working with a block-shortened subcode of a very large code as described in Section 3.2. This idea also build upon the work of Wieschebrink 29] who proved that deciding whether a code is equivalent to a shortened code is NP-complete. In our case, the result is to hide the Cauchy structure of the private code in a general dyadic structure, rather than disguising a quasi-cyclic code as another one with the same symmetry.

We now give a reduction of the problem of decoding the particular class of quasi-dyadic codes to the well-studied syndrome decoding problem, classical in coding theory and known to be NP-complete [4].

Definition 8 (Syndrome decoding). Let $\mathbb{F}_{q}$ be a finite field, and let $(H, w, s)$ be a triple consisting of a matrix $H \in \mathbb{F}_{q}^{r \times n}$, an integer $w<n$, and a vector 
$s \in \mathbb{F}_{q}^{r}$. Does there exist a vector $e \in \mathbb{F}_{q}^{n}$ of Hamming weight wt $(e) \leqslant w$ such that $H e^{\top}=s^{\top}$ ?

The corresponding problem for quasi-dyadic matrices reads:

Definition 9 (Quasi-Dyadic Syndrome Decoding). Let $\mathbb{F}_{q}$ be a finite field, and let $(H, w, s)$ be a triple consisting of a quasi-dyadic matrix $H \in \Delta\left(\mathbb{F}_{q}^{\ell}\right)^{r \times n}$, an integer $w<\ell n$, and a vector $s \in \mathbb{F}_{q}^{\ell r}$. Does there exist a vector $e \in \mathbb{F}_{q}^{\ell n}$ of Hamming weight wt $(e) \leqslant w$ such that $H e^{\top}=s^{\top}$ ?

Theorem 4. The quasi-dyadic syndrome decoding problem (QD-SDP) is polynomially equivalent to the syndrome decoding problem (SDP). In other words, decoding quasi-dyadic codes is as hard in the worst case as decoding general codes.

Proof. The QD-SDP, being an instance of the SDP restricted to a particular class of codes, is clearly a decision problem in NP.

Consider now a generic instance $\left(H^{\prime}, w^{\prime}, s^{\prime}\right) \in \mathbb{F}_{q}^{r \times n} \times \mathbb{Z} \times \mathbb{F}_{q}^{r}$ of the SDP. Assume one is given an oracle that solves the QD-SDP over $\Delta\left(\mathbb{F}_{q}^{\ell}\right)$ for some given $\ell>0$. Let $v_{\ell} \in \mathbb{F}_{q}^{\ell}$ be the all-one vector, i.e. $\left(v_{\ell}\right)_{j}=1$ for all $j$. Define the quasi-dyadic matrix $H=H^{\prime} \otimes I_{\ell} \in \Delta\left(\mathbb{F}_{q}^{\ell}\right)^{r \times n}$ with blocks $H_{i j}=H_{i j}^{\prime} I_{\ell}$, the vector $s=s^{\prime} \otimes v_{\ell} \in\left(\mathbb{F}_{q}^{\ell}\right)^{r}$ with blocks $s_{i}=s_{i}^{\prime} v_{\ell}$, and $w=\ell w^{\prime}$. It is evident that the instance $(H, w, s) \in \Delta\left(\mathbb{F}_{q}^{\ell}\right)^{r \times n} \times \mathbb{Z} \times\left(\mathbb{F}_{q}^{\ell}\right)^{r}$ of the QD-SDP can be constructed in polynomial time.

Assume now that there exists $e \in \mathbb{F}_{q}^{\ell n}$ of Hamming weight wt $(e) \leqslant w$ such that $H e^{\top}=s^{\top}$. For all $0 \leqslant i<\ell$, let $e_{i}^{\prime} \in \mathbb{F}_{q}^{n}$ be the vector with elements $\left(e_{i}^{\prime}\right)_{j}=e_{i+j \ell}, 0 \leqslant j<n$, so that the $e_{i}^{\prime}$ are interleaved to compose $e$. Obviously at least one of the $e_{i}^{\prime}$ has Hamming weight not exceeding $w / \ell=w^{\prime}$, and by the construction of $H$ any of them satisfies $H e_{i}^{\prime \top}=s^{\prime \top}$, constituting a solution to the given instance of the SDP. This effectively reduces the SDP to the QD-SDP for any given $\ell$ in polynomial time. Thus, the QD-SDP itself is NP-complete.

Although this theorem does not say anything about hardness in the average case, it nevertheless strengthens our claim that the family of codes we propose is in principle no less suitable for cryptographic applications than a generic code, in the sense that, should the QD-SDP problem turn out to be feasible in the worst case, then all coding-based cryptosystems would definitely be ruled out, regardless of which code is used to instantiate them. Incidentally, the expected running time of all known algorithms for the SDP (and the QD-SDP) is exponential, so there is empirical evidence that the average case is also very hard. We stress, however, that particular cryptosystems based on quasi-dyadic codes will usually depend on more specific security assumptions, whose assessment transcends the scope of this paper.

\section{$5 \quad$ Efficiency Considerations}

Due to their simple structure the matrices in our proposal can be held on a simple vector not only for long-term storage or transmission, but for processing as well. 
The operation of multiplying a vector by a (quasi-)dyadic matrix is at the core of McEliece encryption. The fast Walsh-Hadamard transform (FWHT) 12 approach for dyadic convolution via lifting 2 to characteristic 0 leads to the asymptotic complexity $O(n \lg n)$ for this operation and hence also for encoding. Sarwate's decoding method [24] sets the asymptotic cost of that operation at roughly $O(n \lg n)$ as well for the typical cryptographic setting $t=O(n / \lg n)$.

Inversion, on the other hand, can be carried out in $O(n)$ steps: one can show by induction that a binary dyadic matrix $\Delta(h)$ of dimension $n$ satisfies $\Delta^{2}=$ $\left(\sum_{i} h_{i}\right)^{2} I$, and hence its inverse, when it exists, is $\Delta^{-1}=\left(\sum_{i} h_{i}\right)^{-2} \Delta$, which can be computed in $O(n)$ steps since it is entirely determined by its first row.

Converting a quasi-dyadic matrix to systematic (echelon) form involves a Gaussian elimination incurring about $d^{2} \ell$ products of dyadic $t \times t$ submatrices, implying a complexity $O\left(d^{2} \ell t \lg t\right)=O\left(d^{2} n \lg n\right)$, and hence the overall cost of formatting is $O(n \lg n)$ as long as $d$ is a small constant, which is indeed the case in practice since maximum size reduction is achieved when $\mathbb{F}_{p}$ is a large proper subfield of $\mathbb{F}_{q}$ (see Section 5.1). Notice that, contrary to systems based on quasi-circulant matrices [8, Proposition 3.4], our proposal does not require a lengthy process, involving expensive $O\left(n^{3}\right)$ matrix rank computations to construct a generator matrix in suitable form, often larger than one would expect for a code of the given dimension.

Table 1 summarizes the asymptotic complexities of code generation (mainly due to systematic formatting), encoding and decoding, which coincide with the complexities of key generation, encryption and decryption of typical cryptosystems based on codes.

Table 1. Operation complexity relative to the code length $n$

\begin{tabular}{|ccc|}
\hline operation & generic & ours \\
\hline Code generation & $O\left(n^{3}\right)$ & $O(n \lg n)$ \\
Encode/Decode & $O\left(n^{2}\right)$ & $O(n \lg n)$ \\
\hline
\end{tabular}

\subsection{Suggested Parameters}

Several trade-offs are possible when choosing parameters for a particular application. One may wish to minimize the key size, or increase speed, or simplify the underlying arithmetic, or attaining a balance between them. We present here some non-exhaustive combinations. The number of errors is always a power of 2 to enable maximum size reduction.

Table 2 shows the influence of varying the subfield degree while keeping fixed the approximate security level and the number of design errors. In general, codes over larger subfields allow for smaller keys as already indicated in [3]. For these parameters the number of possible codes ranges from $2^{392}$ to $2^{731}$.

${ }^{2}$ We are grateful to Dan Bernstein for suggesting the lifting technique to emulate the FWHT in characteristic 2 . 
Table 2. Sample parameters for a fixed number of errors $(t=128)$ and approximately 128-bit security level, using a subcode over the subfield $\mathbb{F}_{2^{s}}$ of $\mathbb{F}_{2^{16}}$

\begin{tabular}{|lccc|}
\hline$s$ & $n$ & $k$ & size (bits) \\
\hline 1 & 4096 & 2048 & 32768 \\
2 & 2560 & 1536 & 24576 \\
4 & 1408 & 896 & 14336 \\
8 & 768 & 512 & 8192 \\
\hline
\end{tabular}

Table 3 displays a different trade-off whereby the key size and the subfield are kept constant at the cost of varying the number of errors and the code length. The estimated security level on column 'level' refers to the approximate logarithmic cost of the best known attack according to the guidelines in [7].

Table 3. Sample parameters for a fixed key size (8192 bits, corresponding to $k=512$ ), using a subcode over the subfield $\mathbb{F}_{2^{8}}$ of $\mathbb{F}_{2^{16}}$

\begin{tabular}{|ccc|}
\hline$n$ & $t$ & level \\
\hline 640 & 64 & 102 \\
768 & 128 & 136 \\
1024 & 256 & 168 \\
\hline
\end{tabular}

One more trade-off is obtained by defining the subfield subcode over the base field itself, following the common practice for generic codes. The corresponding settings 3 are summarised on Table 4.

Table 4. Sample parameters for a subcode over the base subfield $\mathbb{F}_{2}$ of $\mathbb{F}_{2^{16}}$

\begin{tabular}{|ccccc|}
\hline level & $n$ & $k$ & $t$ & size (bits) \\
\hline 80 & 2304 & 1280 & 64 & 20480 \\
112 & 3584 & 1536 & 128 & 24576 \\
128 & 4096 & 2048 & 128 & 32768 \\
192 & 7168 & 3072 & 256 & 49152 \\
256 & 8192 & 4096 & 256 & 65536 \\
\hline
\end{tabular}

Table 5 contains a variety of balanced parameters for practical security levels. Although we do not recommend these for actual deployment before further analysis is carried out, these parameters were chosen to stress the possibilities of our proposal while giving a realistic impression of what one might indeed

${ }^{3}$ The actual security levels computed according to the attack strategy in [7] for the parameters suggested in Table 4 are, respectively, 84.3, 112.3, 136.5, 216.0, and 265.1. We are grateful to Christiane Peters for kindly providing these estimates. 
adopt in practice. The target security level, roughly corresponding to the estimated logarithmic cost of the best known attack according to the guidelines in [7, is shown on the 'level' column. The 'size' column contains the amount of bits effectively needed to store a quasi-dyadic generator or parity-check matrix in systematic form. The size of a corresponding systematic matrix for a generic Goppa code at roughly the same security level as suggested in [7] is given on column 'generic'. The 'shrink' column contains the size ratio between such a generic matrix and a matching quasi-dyadic matrix. The 'RSA' column lists the typical size of a (quantum-susceptible) RSA modulus at the specified security level (more accurate RSA estimates can be found in 2021]). To assess our results against what can be achieved by other post-quantum settings, column ' $Q \mathrm{C}$ ' lists key sizes for quasi-cyclic codes of approximately the specified security level (although not necessarily for the same code length, dimension, and distance) as suggested in [3], column 'LDPC' does the same for (quasi-cyclic) low-density parity-check codes as discussed in [2, and finally the 'NTRU' column contains the range (from size-optimal to speed-optimal) of NTRU key sizes as suggested in the draft IEEE 1363.1 standard [13. For these very compact parameters the number of possible codes ranges between $2^{346}$ and $2^{392}$, less than those of Table 2 but still very large.

Table 5. Sample parameters for a subcode over the subfield $\mathbb{F}_{2^{8}}$ of $\mathbb{F}_{2^{16}}$

\begin{tabular}{|ccccccccccc|}
\hline level & $n$ & $k$ & $t$ & size & generic & shrink & RSA & QC & LDPC & NTRU \\
\hline 80 & 512 & 256 & 128 & 4096 & 460647 & 112 & 1024 & 6750 & 49152 & - \\
112 & 640 & 384 & 128 & 6144 & 1047600 & 170 & 2048 & 14880 & - & $4411-7249$ \\
128 & 768 & 512 & 128 & 8192 & 1537536 & 188 & 3072 & 20400 & - & $4939-8371$ \\
192 & 1280 & 768 & 256 & 12288 & 4185415 & 340 & 7680 & - & - & $7447-11957$ \\
256 & 1536 & 1024 & 256 & 16384 & 7667855 & 468 & 15360 & - & - & $11957-16489$ \\
\hline
\end{tabular}

For the parameters on Table 5, we observed the timings on Table 6 (measured in $\mathrm{ms}$ ) for generic Goppa codes and quasi-dyadic (QD) codes, and also for RSA to assess the efficiency relative to a very common pre-quantum cryptosystem. We made no serious attempt at optimizing the implementation, which was done in $\mathrm{C}++$ and tested on an AMD Turion 64X2 2.4 GHz. Benchmarks for RSA-15360 were omitted due to the enormous time needed to generate suitable parameters.

Table 6. Benchmarks for typical parameters

\begin{tabular}{|cccccccccccc|}
\hline level & \multicolumn{3}{c}{ generation } & & \multicolumn{3}{c}{ encoding } & & \multicolumn{3}{c|}{ decoding } \\
\cline { 2 - 3 } \cline { 9 - 11 } & RSA & generic & QD & & RSA & generic & QD & & RSA & generic & QD \\
\hline 80 & 563 & 375 & 17.2 & & 0.431 & 0.736 & 0.817 & & 15.61 & 1.016 & 3.685 \\
112 & 1971 & 1320 & 18.7 & & 1.548 & 1.696 & 1.233 & & 110.34 & 2.123 & 4.463 \\
128 & 4998 & 2196 & 20.5 & & 3.467 & 2.433 & 1.575 & & 349.91 & 3.312 & 5.261 \\
192 & 628183 & 13482 & 47.6 & 22.320 & 6.872 & 4.695 & & 5094.10 & 8.822 & 17.783 \\
256 & - & 27161 & 54.8 & & - & 12.176 & 6.353 & & - & 15.156 & 21.182 \\
\hline
\end{tabular}




\section{Conclusion and Further Research}

We have described how to generate Goppa codes in quasi-dyadic form suitable for cryptographic applications. Key sizes for a typical McEliece-like cryptosystem are roughly a factor $t=\tilde{O}(n)$ smaller than generic Goppa codes, and keys can be kept in this compact size not only for storing and transmission but for processing as well. In the binary case these codes can correct the full design number of errors. This brings the size of cryptographic keys to within a factor 4 or less of equivalent RSA keys, comparable to NTRU keys. Our work provides an alternative to conventional cyclic and quasi-cyclic codes, and benefits from the same trapdoor-hiding techniques proposed by Wieschebrink in general [29], and by Berger et al. for that family of codes [3].

The complexity of all operations in McEliece and related cryptosystems is reduced to $O(n \lg n)$. Other cryptosystems can also benefit from dyadic codes, e.g. entity identification and certain digital signatures for which double circulant codes have been proposed [9] could use dyadic codes instead, even random ones without a Goppa trapdoor. One further line of research is whether one can securely combine the techniques in 22 with ours to define quasi-dyadic, low-density parity-check (QD-LDPC) codes that are suitable for cryptographic purposes and potentially even shorter than plain quasi-dyadic codes.

Interestingly, it is equally possible to define lattice-based cryptosystems with short keys using dyadic lattices entirely analogous to ideal (cyclic) lattices as proposed by Micciancio [17, and achieving comparable size reduction. We leave this line of inquiry for future research since it falls outside the scope of this paper.

\section{Acknowledgments}

We are most grateful and deeply indebted to Marco Baldi, Dan Bernstein, PierreLouis Cayrel, Philippe Gaborit, Steven Galbraith, Robert Niebuhr, Christiane Peters, Nicolas Sendrier, and the anonymous reviewers for their valuable comments and feedback during the preparation of this work.

\section{References}

1. Baldi, M., Chiaraluce, F.: Cryptanalysis of a new instance of McEliece cryptosystem based on QC-LDPC code. In: IEEE International Symposium on Information Theory - ISIT 2007, Nice, France, pp. 2591-2595. IEEE, Los Alamitos (2007)

2. Baldi, M., Chiaraluce, F., Bodrato, M.: A new analysis of the mcEliece cryptosystem based on QC-LDPC codes. In: Ostrovsky, R., De Prisco, R., Visconti, I. (eds.) SCN 2008. LNCS, vol. 5229, pp. 246-262. Springer, Heidelberg (2008)

3. Berger, T.P., Cayrel, P.-L., Gaborit, P., Otmani, A.: Reducing key length of the McEliece cryptosystem. In: Preneel, B. (ed.) AFRICACRYPT 2009. LNCS, vol. 5580, pp. 77-97. Springer, Heidelberg (2009), http://www.unilim.fr/pages_perso/philippe.gaborit/reducing.pdf 
4. Berlekamp, E., McEliece, R., van Tilborg, H.: On the inherent intractability of certain coding problems. IEEE Transactions on Information Theory 24(3), 384386 (1978)

5. Bernstein, D.J.: List decoding for binary Goppa codes (2008) (preprint), http://cr.yp.to/papers.html\#goppalist

6. Bernstein, D.J., Buchmann, J., Dahmen, E.: Post-Quantum Cryptography. Springer, Heidelberg (2008)

7. Bernstein, D.J., Lange, T., Peters, C.: Attacking and defending the mcEliece cryptosystem. In: Buchmann, J., Ding, J. (eds.) PQCrypto 2008. LNCS, vol. 5299, pp. 31-46. Springer, Heidelberg (2008), http://www. springerlink. com/content/68v69185x478p53g

8. Gaborit, P.: Shorter keys for code based cryptography. In: International Workshop on Coding and Cryptography - WCC 2005, Bergen, Norway, pp. 81-91. ACM Press, New York (2005)

9. Gaborit, P., Girault, M.: Lightweight code-based authentication and signature. In: IEEE International Symposium on Information Theory - ISIT 2007, Nice, France, pp. 191-195. IEEE, Los Alamitos (2007)

10. Gibson, J.K.: Severely denting the Gabidulin version of the McEliece public key cryptosystem. Designs, Codes and Cryptography 6(1), 37-45 (1995)

11. Gibson, J.K.: The security of the Gabidulin public key cryptosystem. In: Maurer, U.M. (ed.) EUROCRYPT 1996. LNCS, vol. 1070, pp. 212-223. Springer, Heidelberg (1996)

12. Gulamhusein, M.N.: Simple matrix-theory proof of the discrete dyadic convolution theorem. Electronics Letters 9(10), 238-239 (1973)

13. IEEE P1363 Working Group. IEEE 1363-1: Standard Specifications for Public-Key Cryptographic Techniques Based on Hard Problems over Lattices, Draft (2009), http://grouper.ieee.org/groups/1363/lattPK/index.html

14. Loidreau, P., Sendrier, N.: Some weak keys in McEliece public-key cryptosystem. In: IEEE International Symposium on Information Theory - ISIT 1998, Boston, USA, p. 382. IEEE, Los Alamitos (1998)

15. MacWilliams, F.J., Sloane, N.J.A.: The theory of error-correcting codes. NorthHolland Mathematical Library, vol. 16 (1977)

16. McEliece, R.: A public-key cryptosystem based on algebraic coding theory. The Deep Space Network Progress Report, DSN PR 42-44 (1978),

http://ipnpr.jpl.nasa.gov/progressreport2/42-44/44N.PDF

17. Micciancio, D.: Generalized compact knapsacks, cyclic lattices, and efficient oneway functions. Computational Complexity 16(4), 365-411 (2007)

18. Monico, C., Rosenthal, J., Shokrollahi, A.: Using low density parity check codes in the McEliece cryptosystem. In: IEEE International Symposium on Information Theory - ISIT 2000, Sorrento, Italy, p. 215. IEEE, Los Alamitos (2000)

19. Niederreiter, H.: Knapsack-type cryptosystems and algebraic coding theory. Problems of Control and Information Theory 15(2), 159-166 (1986)

20. European Network of Excellence in Cryptology (ECRYPT). ECRYPT yearly report on algorithms and keysizes (2007-2008). D.SPA.28 Rev. 1.1, IST-2002-507932 ECRYPT, 07/2008 (2008),

http://www.ecrypt.eu.org/ecrypt1/documents/D.SPA.28-1.1.pdf

21. National Institute of Standards and Technology (NIST). Recommendation for key management - part 1: General (2007),

http://csrc.nist.gov/publications/nistpubs/800-57/

sp800-57-Part1-revised2_Mar08-2007.pdf 
22. Otmani, A., Tillich, J.-P., Dallot, L.: Cryptanalysis of two McEliece cryptosystems based on quasi-cyclic codes (2008) (preprint), http://arxiv.org/abs/0804.0409v2

23. Patterson, N.J.: The algebraic decoding of Goppa codes. IEEE Transactions on Information Theory 21(2), 203-207 (1975)

24. Sarwate, D.V.: On the complexity of decoding Goppa codes. IEEE Transactions on Information Theory 23(4), 515-516 (1977)

25. Schechter, S.: On the inversion of certain matrices. Mathematical Tables and Other Aids to Computation 13(66), 73-77 (1959), http://www.jstor.org/stable/2001955

26. Sendrier, N.: Finding the permutation between equivalent linear codes: the support splitting algorithm. IEEE Transactions on Information Theory 46(4), 1193-1203 (2000)

27. Sidelnikov, V., Shestakov, S.: On cryptosystems based on generalized ReedSolomon codes. Discrete Mathematics 4(3), 57-63 (1992)

28. Tzeng, K.K., Zimmermann, K.: On extending Goppa codes to cyclic codes. IEEE Transactions on Information Theory 21, 721-726 (1975)

29. Wieschebrink, C.: Two NP-complete problems in coding theory with an application in code based cryptography. In: IEEE International Symposium on Information Theory - ISIT 2006, Seattle, USA, pp. 1733-1737. IEEE, Los Alamitos (2006) 